\title{
Rotation-invariant fingerprint matching using radon and DCT
}

\author{
SANGITA BHARKAD* and MANESH KOKARE
}

Department of Electronics and Telecommunication, Government College of Engineering, Osmanpura, Aurangabad 431005, India

e-mail: sbharkad@yahoo.co.in; mbkokare@yahoo.com

MS received 26 December 2016; revised 12 May 2017; accepted 2 June 2017; published online 20 November 2017

\begin{abstract}
A new set of promising rotation-invariant features based on radon and discrete cosine transform (DCT) is proposed for fingerprint matching. The radon and DCT of a tiny area in the region of core point of fingerprint image is computed. In the proposed method only 34\% DCT coefficients are used for feature extraction. Competency of this approach is tested on standard databases, namely FVC2002 and FVC2004. This approach provides $70 \%$ genuine acceptance rate $(\mathrm{GAR})$ at $\sim 0 \%$ false acceptance rate (FAR) and $95 \%$ GAR at $10 \%$ FAR on rotated and non-rotated databases, respectively. Experimental results prove that the proposed feature extraction approach is rotation invariant.
\end{abstract}

Keywords. Biometrics; radon transform; discrete cosine transform; feature extraction; similarity measures.

\section{Introduction}

Biometrics can be defined as recognition of a person based on physiological or behavioural uniqueness. Biometrics is competent enough to consistently discriminate between an authorized person and a pretender. The most vital feature of biometrics is that it cannot be elapsed or misplaced. At the time of identification, the person should be present physically. Conventional knowledge-based and token-based schemes are not reliable and proficient because one can brake these methods easily. Hence biometrics is innately more trustworthy and more capable.

Most popular features used in fingerprint recognition system are minutiae points. These features are computed from the information of end point and bifurcation point of ridges. It is somewhat difficult to extract robust minutiae features in case of translational and rotational distortion of fingerprint images. Spurious minutiae points created due to translational and rotational distortion will spoil performance of fingerprint identification system. The texturebased features of fingerprint image can be extracted using Gabor filter, discrete wavelet transform, ridgelets and curvelets. These texture-based techniques are unable to tackle the rotational distortion of fingerprint images. Hence the approach robust to rotational distortion of fingerprint images is the need of reliable fingerprint identification system.

*For correspondence

\subsection{Motivation}

Uniqueness and perseverance are two fundamental properties of fingerprint identification. Perseverance is defined as the important characteristics of fingerprints that do not alter with time. Uniqueness is defined as the fingerprint that is distinctive to a human being as elaborated in [1]. The strength of the perseverance has been recognized by the anatomy and morphogenesis of friction ridge skin. Uniqueness has been acknowledged to be correct based on experimental results.

Fingerprint matching using the local features of fingerprint image is very popular. Ridge ending and ridge bifurcation points are the local features of fingerprint image. The accuracy of minutia-based methods totally depends on the extraction of genuine ridge ending and ridge bifurcation points. These points are also called as minutiae features. For noisy images, it is not easy to locate true finer points like ridge ending and ridge bifurcation. Poor quality image generates spurious minutiae points, which affects accuracy. Jain et al [2] proposed a method that extracts texture-based features of fingerprint image using the Gabor filter. This algorithm defeats the constraints of minutiabased feature extraction approaches. This texture-based method is capable of exploring the small as well as significant information of fingerprint image. The advantage of texture-based approaches is that it gives good accuracy However, the main drawback of Gabor-based method is its high computational complexity due to the non-orthogonal basis functions. Hence, Gabor-based method requires large memory as well as more matching time. These drawbacks of Gabor-filter-based method can be overcome using DWT- 


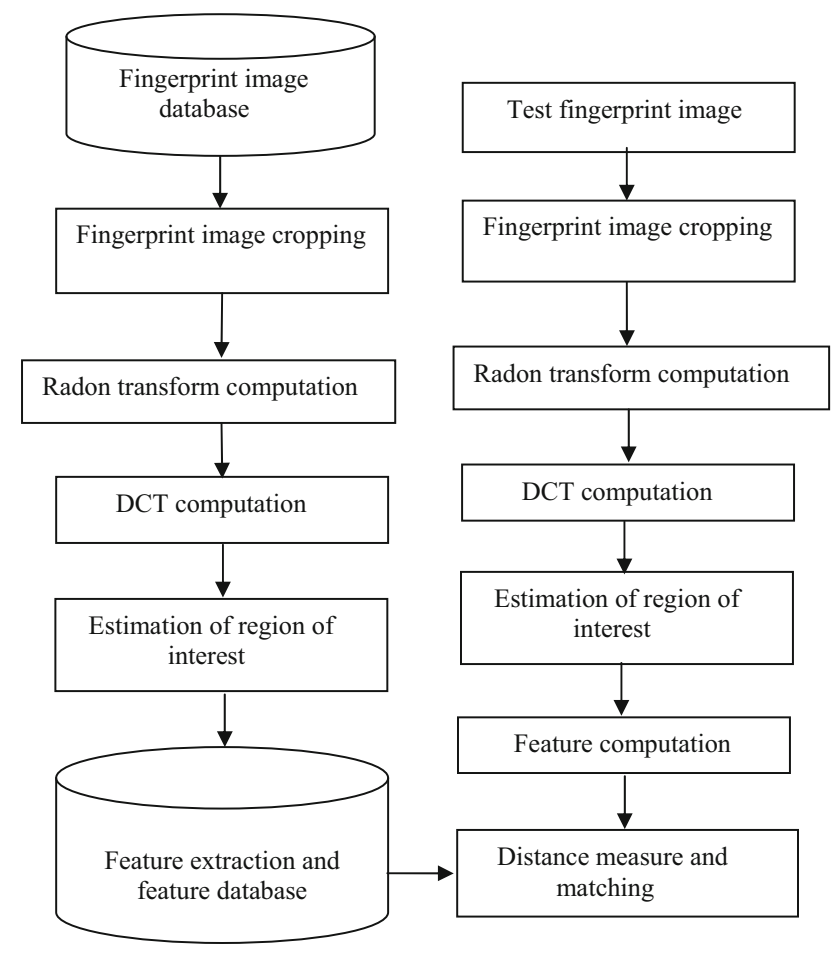

Figure 1. Flow design of the proposed method.

based feature extraction methods [3-5] as DWT is orthogonal. However, accuracy of DWT-based feature extraction methods is not promising. This is the limitation of DWT-based feature extraction methods.

To achieve good accuracy with low computational complexity, we used the discrete cosine transform (DCT)-based features for fingerprint matching. DCT-based features gives good recognition rate on the database available at Bologna University but the performance is poor on FVC2002 and FVC2004 databases because in these databases most of the images have rotation less than or greater than $45^{\circ}$. DCT is not a rotation-invariant transform. Existing feature extraction methods based on Gabor filter and DWT are also not rotation invariant. In the texture-based feature extraction method proposed by Jain et al [2], texture features called finger code of fingerprint image extracted using the Gabor filter are rotated at time of matching.

In this research work our main goal is to develop the rotation-invariant good-quality set of features with compact representation without compromising on the accuracy. The novelty of proposed method is that it extracts the rotationinvariant features using radon transform and translation invariance is achieved using DCT.

\subsection{Related work}

There are several fingerprint matching algorithms available in literature, which are based on local features. These local features are assumed to be mainly consistent as illustrated in [6-8]. A computationally uncomplicated fingerprint verification system based on only location of minutiae points in an image is discussed in [9]. Chu and Chiu [10] encoded neighbourhood structure of each minutiae using a new disk structure. This new disk structure lucidly demonstrates the allocation of surrounding minutiae and trains the surrounding minutiae very competently. Jiang et al [11] introduced a convolutional neural-network-based approach for automatic extraction of minutiae points from fingerprint image. This approach does not utilize any preprocessing algorithm such as binarization, thinning and enhancement. This is the main feature of this approach.

However, matching of two feature vectors of minutiae points is not an easy assignment. The minutiae features give only local information of fingerprint image. This local information is not sufficient for fingerprint matching. The quality of minutiae points depends on the merit of image. The challenging job is to compute the genuine minutiae points from degraded finger image. Nagaty [12] computed energy features for fingerprint matching. This features approach performs better than minutia-based approach. Jain et al [2, 13] introduced a bank of filters to obtain information at different orientations for fingerprint matching. This algorithm overcomes the limitations of conventional minutia-based approaches. Also these banks of filters oriented in different directions are sufficient to acquire both fine and coarse information in a fingerprint image. Ross et al [14] adopted both minutiae and directional features of fingerprint image for fingerprint matching. This approach improved the fingerprint matching performance. The performance of hybrid approach is better than individual performance of minutiae and Gabor filter. However, the hybrid fingerprint matching systems are computationally expensive. Fingerprint recognition based on a set of even Gabor filters is introduced in [13, 14]. Horton et al [15] make use of a complex Gabor filter to explore the odd and even features of fingerprint image. Munir and Javed [16] utilized 16 Gabor filters oriented in different directions to extract a good set of features from fingerprint image. The use of more number of Gabor filters raises the accuracy but simultaneously it also intensifies the redundancy and computational complexity, since Gabor bases are nonorthogonal. Jain et al [2] utilized eight Gabor filters with different orientations to extract the features which yielded a feature vector of length 640. Feature vector length of the method proposed by Munir and Javed [16] using 16 Gabor filters is 2048. Bharkad and Kokare [3-5] explored the wavelet domain features using $M$ band, discrete wavelet packet transform and rotated wavelet filters for fingerprint matching. Frequency domain approach based on real valued Hartley transform is discussed in [17]. The computational complexity of wavelet-based method is low as wavelet bases are orthogonal. However, the accuracy of wavelet-based method is not consequently good.

Neural network and random forest classifier were used for fingerprint recognition in [18, 19]. Gu et al [20] 


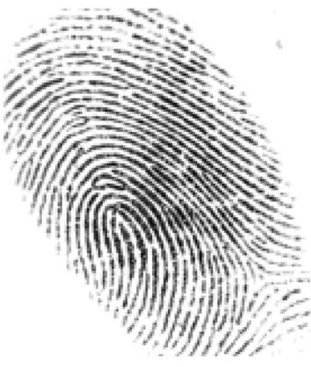

(a)

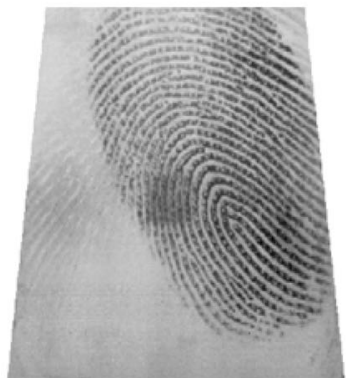

(f)

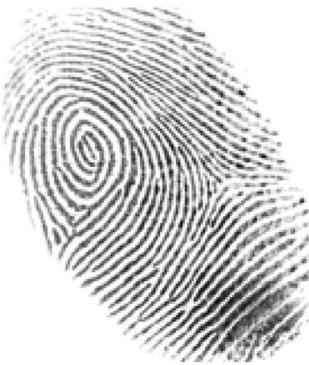

(b)

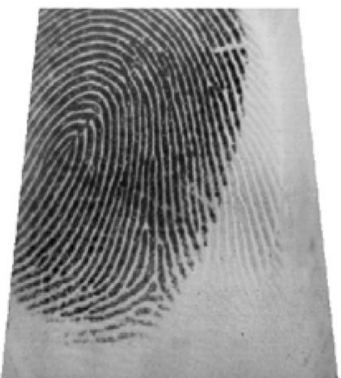

(g)

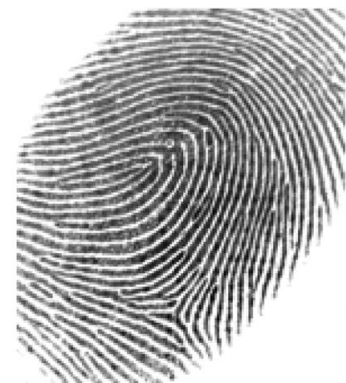

(c)

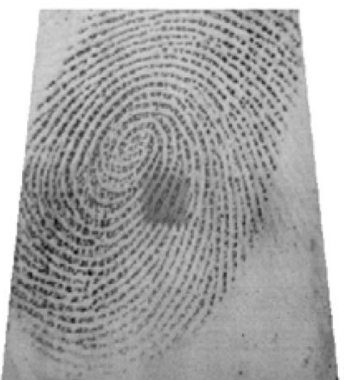

(h)

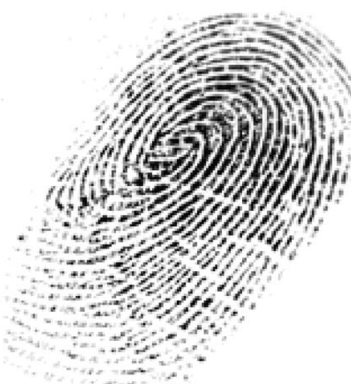

(d)

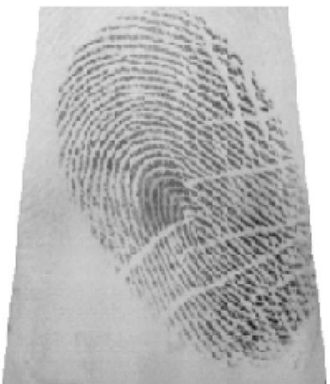

(i)

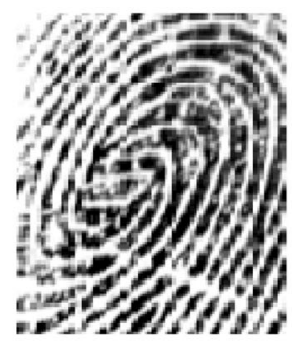

(e)

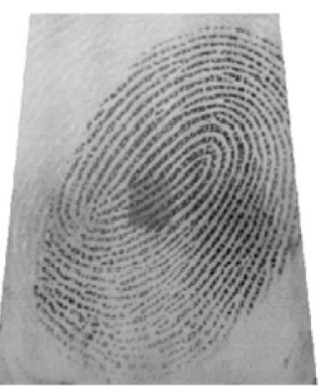

(j)

Figure 2. (a)-(d) Sample images in Database A. (f)-(j) Sample images in Database B. (e) Cropped image of size $120 \times 120$ around core point.

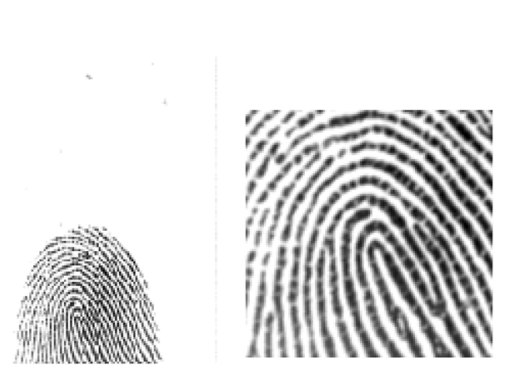

(a)

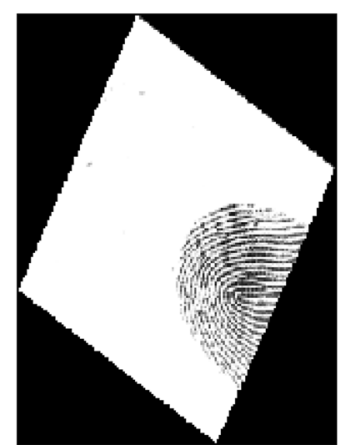

(g)

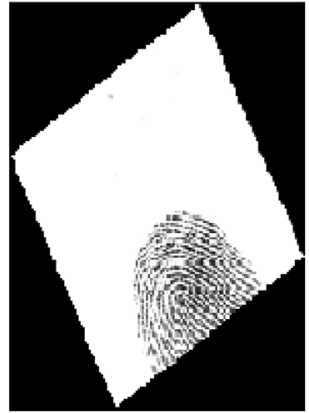

(c)

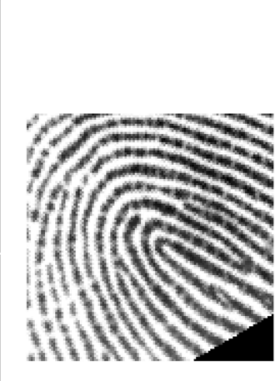

(d)

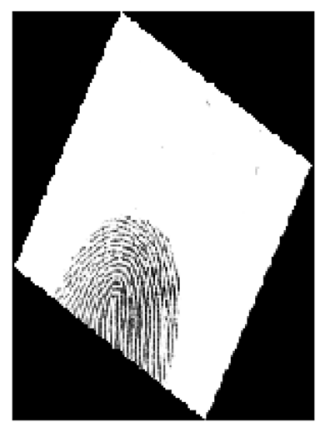

(e)

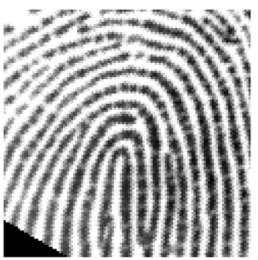

(f)

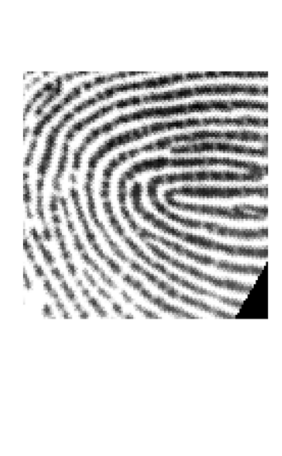

(h)

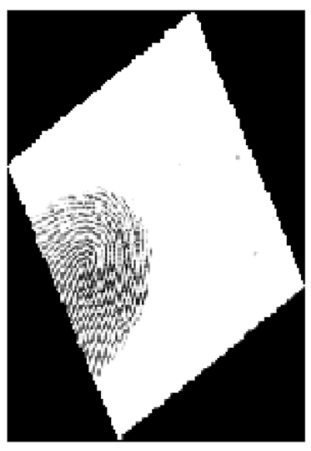

(i)

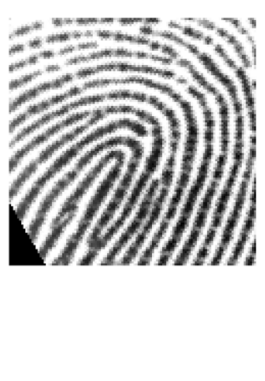

(j)

Figure 3. (a) Original image, (b) cropped original image, (c) original image rotated by $30^{\circ}$, (d) $30^{\circ}-$ rotated cropped image, (e) original image rotated by $-30^{\circ}$, (f) $-30^{\circ}$ - rotated cropped image, (g) original image rotated by $60^{\circ}$, (h) $60^{\circ}-$ rotated cropped image, (i) original image rotated by $-60^{\circ}$ and $(\mathbf{j})-60^{\circ}$ - rotated cropped image. 


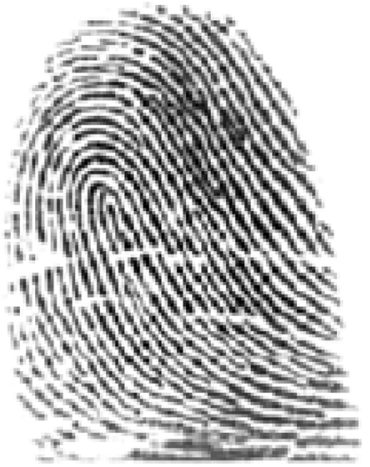

(a)

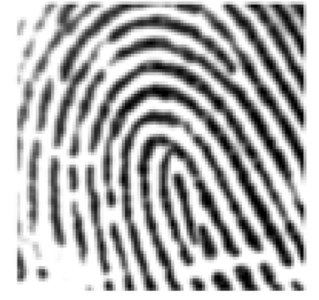

(b)

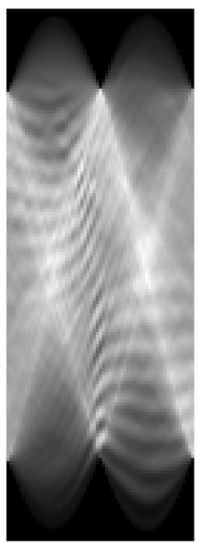

(g)

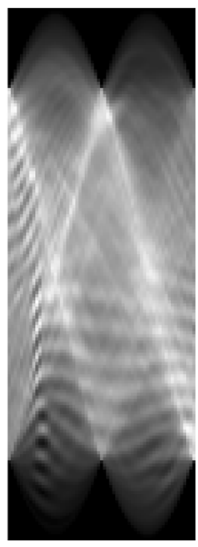

(c)

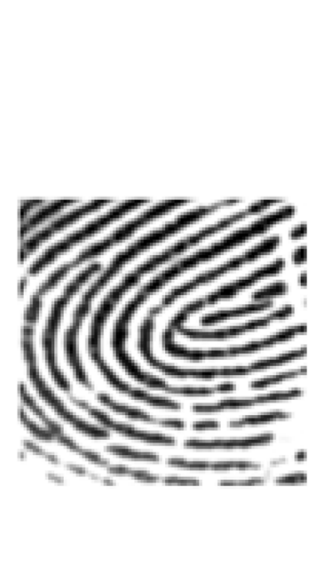

(h)

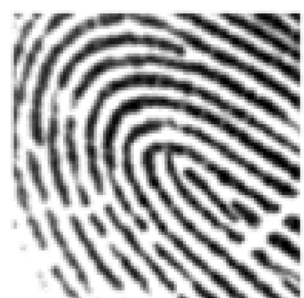

(d)

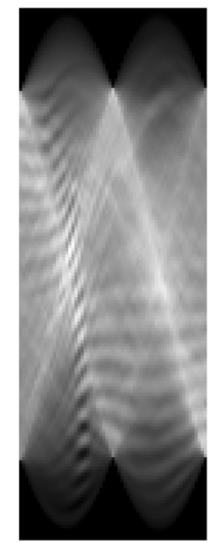

(e)

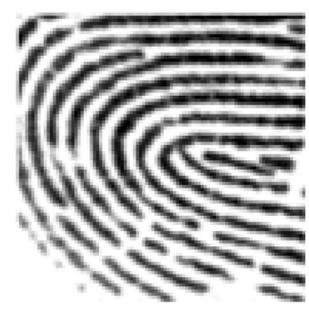

(f)

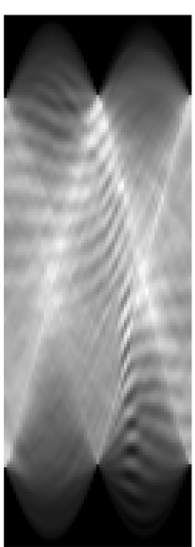

(i)

Figure 4. (a) Original image in FVC2002 Db1_a, (b) cropped original image, (c) radon transform of (b), (d) $30^{\circ}$ - rotated and cropped image, (e) radon transform of (d), (f) $60^{\circ}$ - rotated and cropped image, (g) radon transform of (f), (h) $90^{\circ}-$ rotated and cropped image and (i) radon transform of (h).

discussed a promising scheme for biometric score normalization. The problem of leveraging correlations among the scores is rectified by this method. A detailed study of fingerprints of young children is presented by Jain et al [21], which includes the salient features required to uniquely recognize a child and proper age of child to capture the fingerprints with sufficient fidelity for recognition. Ctirad and Christoph [22] presented an attack detection method for fingerprints. Both liveness detection and alteration detection both are handled by this method. This paper gives a comprehensive survey of the various existing fingerprint sensing technologies, methods for unreliable fingerprint sensors, analysis and discussion on the notions and the schemes of the available fingerprint liveness detection algorithms. Practical issues related to surface fingerprint detection were discussed by $\mathrm{Yu}$ et al [23]. In this paper, spectral-domain optical coherence tomography is demonstrated, which is more useful for forensic science in fingerprint detection. Live and fake fingerprints can be detected using local coherence of a given image [24].
Dissimilarity of the scattering in the image gradient field between live and fake fingerprints is used for liveness detection of fingerprints in [24]. The limitations of existing 3D fingerprint technologies are discussed and are addressed in [25]. In this paper, a 3D fingerprint identification system based on a single camera is implemented to build up oversimplified 3D minutiae matching model.

The concept of rotation-invariant features using block orientation of fingerprint image is discussed in [26]. Sandhan et al [27] explained the method based on conceptual radon outline. This scheme is computationally simple as it does not need any length preprocessing steps. It extracts features using global properties of an image and recognition is performed by $l_{1}$-reduction with quadratic limitations. Ray and Dutta [28] proposed rotation-invariant DCT masks features for classification of texture images. Jadhav and Holambe [29] extracted facial features using radon transform and wavelet transform, which are invariant to deviations in facial appearance and elucidation. Ajmera et al [30] adopted radon transform for extraction of 


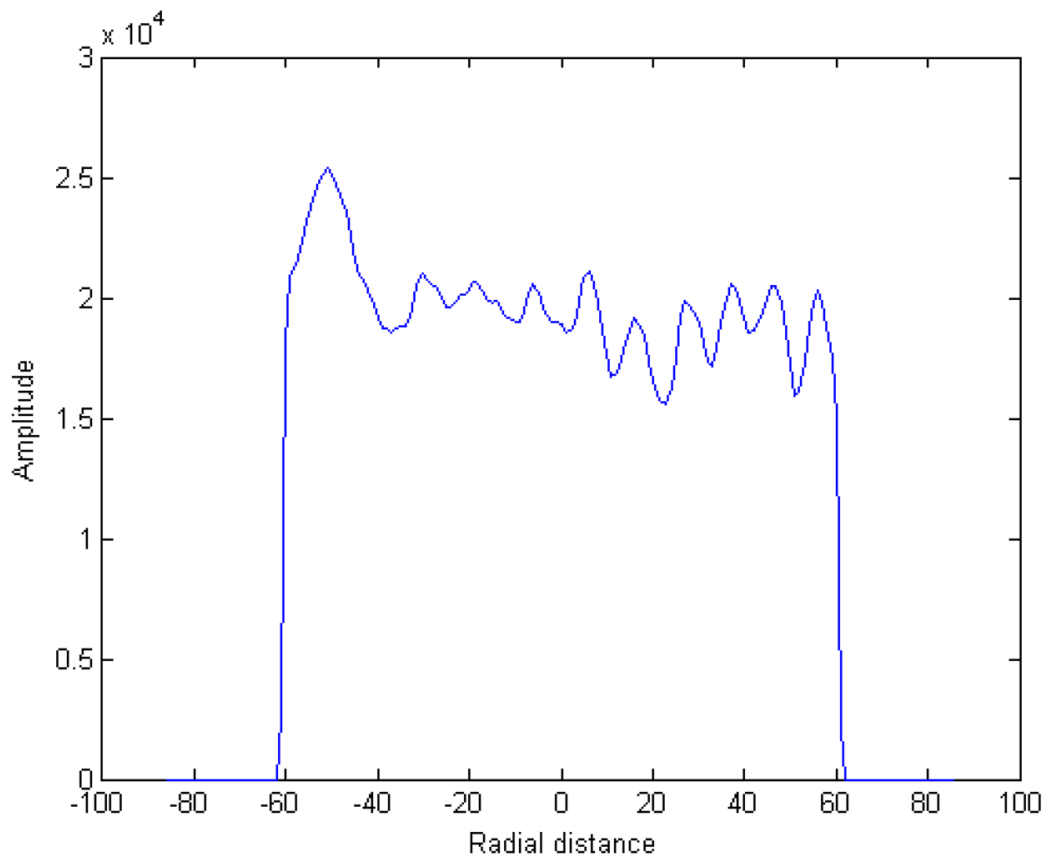

(a)

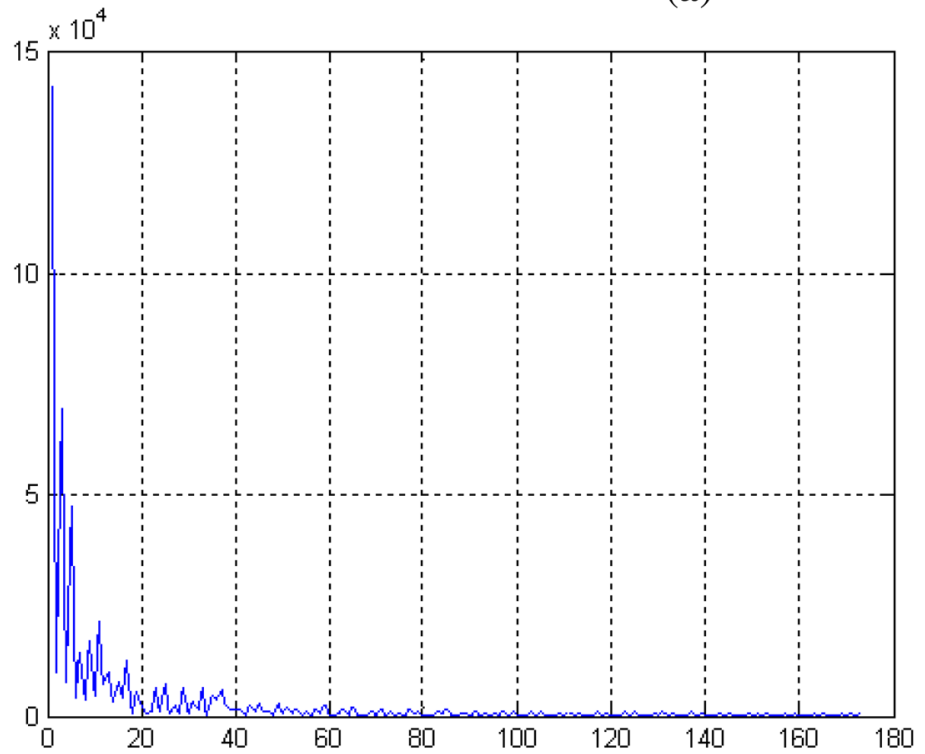

(b)

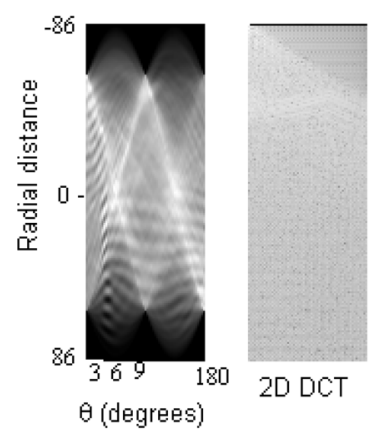

(c) (d)

Figure 5. (a) Radon transform of original cropped image at angle $90^{\circ}$, (b) $1 \mathrm{D}$ DCT of (a), (c) radon transform of original cropped image at 60 different angles between $0^{\circ}$ and $180^{\circ}$ ) and (d) 2D DCT (c).

valuable acoustic features from the speech spectrogram. Dimension of feature vector is reduced using DCT.

\subsection{Our approach}

To pick up the fingerprint matching performance in terms of recognition rate and to trim down the computational complexity we propose a novel rotation-invariant feature extraction method using radon and DCT. The key contributions and novelty of the paper are summarized below.
1. The proposed method extracts features of a small area around the core point of fingerprint image.

2. Rotation invariance is achieved with the help of radon transform, which transfers rotation of an image into corresponding translation in $\theta^{\circ}$. Translation invariance is obtained using DCT. Hence, the proposed method extracts rotation-invariant features. Reduced feature dimensionality is achieved using only $\sim 34 \%$ DCT coefficients to form compact feature vector. This is the key feature of the proposed method. 


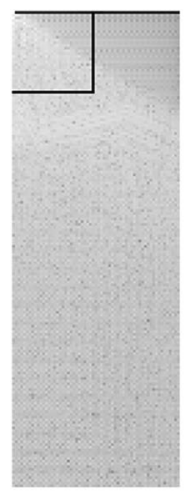

(a)

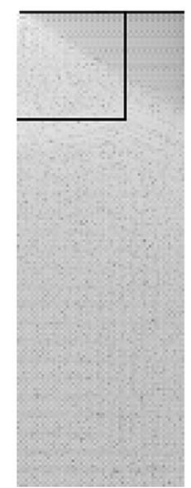

(b)

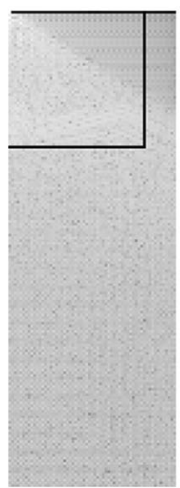

(c)

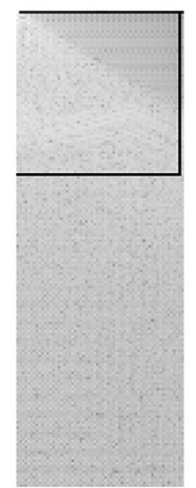

(d)

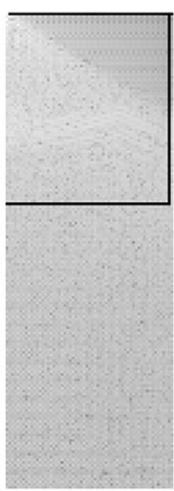

(e)

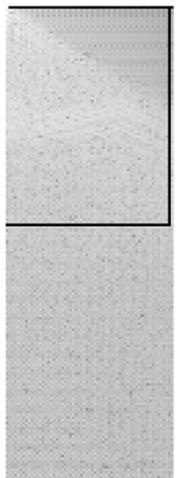

(f)

Figure 6. Six different square portions of 2 D DCT of radon space: (a) $30 \times 30$, (b) $40 \times 40$, (c) $50 \times 50$, (d) $60 \times 60$, (e) $70 \times 70$ and (f) $80 \times 80$.

3. The standard databases, namely FVC2002, FVC2004 and 32 rotated databases derived from $\mathrm{FVC} 2002$ and FVC2004, are used for assessment of performance of this method. It is estimated in terms of genuine acceptance rate (GAR), false acceptance rate (FAR), false rejection rate (FRR) and receiver operating curve (ROC).

4. Results of our approach are compared to those from the existing method based on block orientation features [15], in which the rotation of a fingerprint image is considered while calculating the features. Our approach provides $95 \%$ GAR at 10\% FAR and the method based on block orientation features [15] gives $93 \%$ GAR at $18 \%$ FAR. Hence, the proposed method outperforms over the existing feature extraction method in terms of FAR and GAR.

Rest of the paper is organized as follows. Section 2 gives a brief introduction of radon transform and DCT. Section 3 elaborates on the proposed method. Experimental results are presented in section 4. Section 5 gives conclusions.

\section{Radon transform and DCT}

The radon function acquires several parallel beam projections of the image from different angles by rotating the source in the region of the centre of the image as

Table 1. Comparison of DCT coefficients (\%) in terms of GAR, FAR and FRR on set1.

\begin{tabular}{lcccc}
\hline $\begin{array}{l}\text { Size of square } \\
\text { portions }\end{array}$ & $\begin{array}{c}\text { DCT coefficients } \\
(\sim \%)\end{array}$ & GAR & FAR & FRR \\
\hline $30 \times 30$ & 8 & 75.68 & 26.83 & 24.32 \\
$40 \times 40$ & 15 & 87.28 & 14.33 & 12.72 \\
$50 \times 50$ & 23 & 92 & 10.33 & 8 \\
$60 \times 60$ & 34 & $\mathbf{9 5 . 0 4}$ & $\mathbf{1 0 . 8 6}$ & $\mathbf{4 . 9 6}$ \\
$70 \times 70$ & 39 & 94.6 & 10.35 & 5.4 \\
$80 \times 80$ & 45 & 93.52 & 9.72 & 6.48 \\
\hline
\end{tabular}

demonstrated in [31]. The resulting projections are the addition of the intensities of the pixels in each direction. It transforms a 2D image with lines (line-trends) into a domain of the possible line parameters $\rho$ and $\theta$. The $\rho$ is the smallest distance from the origin and $\theta$ is its angle with the $x$-axis. With the help of $\rho$ and $\theta$, a line can be represented as

$$
\rho=(x \cos \theta+y \sin \theta) .
$$

The radon transform of a 2D image $f(x, y)$ can be defined as

$$
R(\rho, \theta)=\int_{-\infty}^{\infty} \int_{-\infty}^{\infty} f(x, y) \delta(\rho-x \cos \theta-y \sin \theta) d x d y
$$

Robustness to zero-mean white noise is the important property of radon transform. The term image transform refers to a class of unitary matrices used for representing images. The unitary transform consists of orthogonal basis functions. These basis functions help characterize one-dimensional signal in the form orthogonal series of basis functions. An image is able to spread out in terms of discrete set of basis arrays called basis images. Energy conservation, energy compaction and decorrelation are the important properties of these transforms. The two-dimensional DCT [32, 33] of size $M \times N$ image $f(x, y)$ can be represented by the following equation:

$$
\begin{aligned}
C(u, v)= & \alpha(u) \alpha(v) \sum_{x=0}^{M-1} \sum_{y=0}^{N-1} f(x, y) \cos \left(\frac{(2 x+1) u \pi}{2 M}\right) \\
& \cos \left(\frac{(2 y+1) v \pi}{2 N}\right)
\end{aligned}
$$

where $0 \leq u \leq M-1,0 \leq v \leq N-1$. DCT is a real valued, orthogonal and separable transform. It has outstanding energy compaction for highly correlated data. 


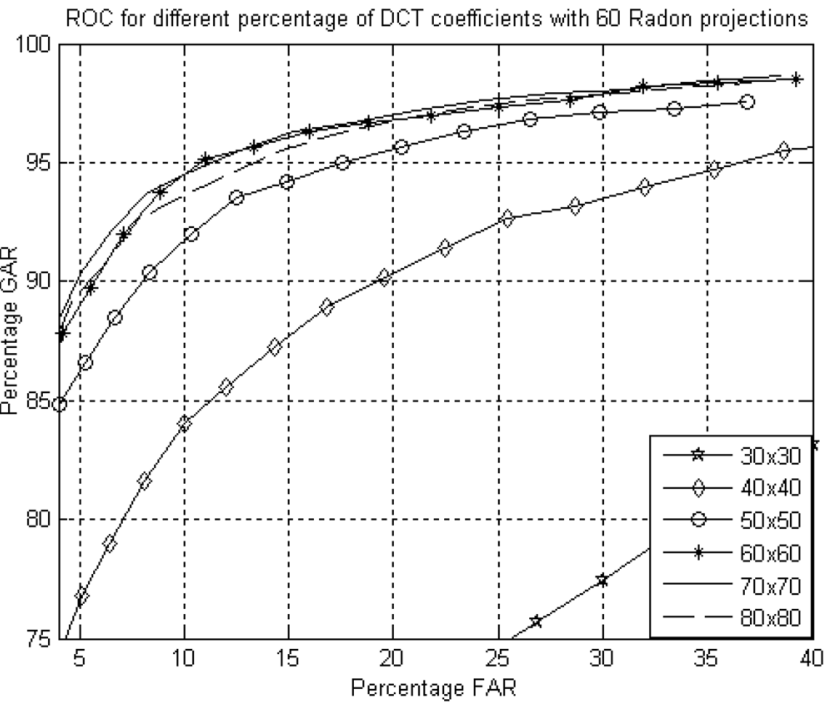

(a)

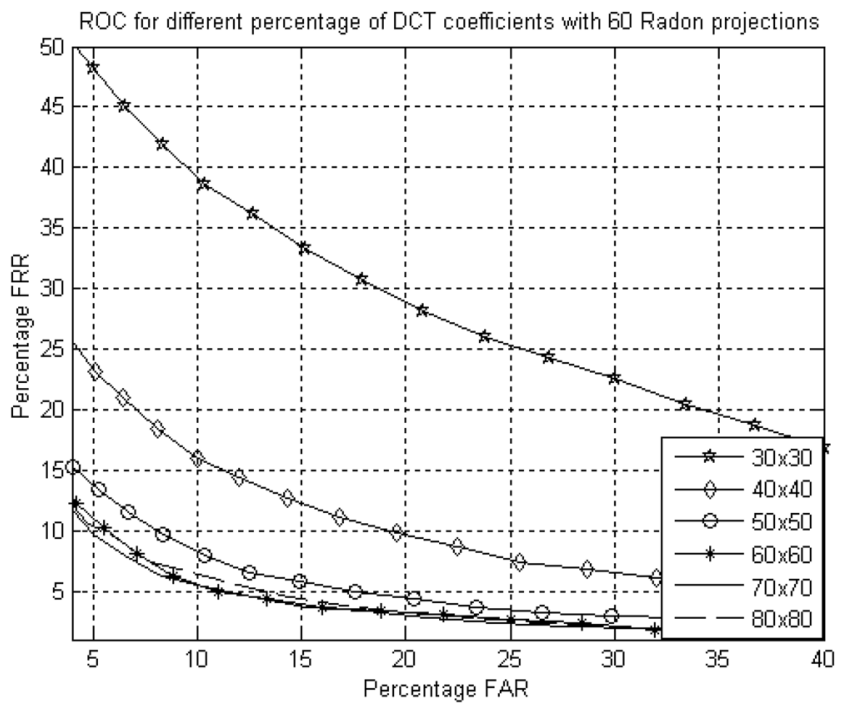

(c)

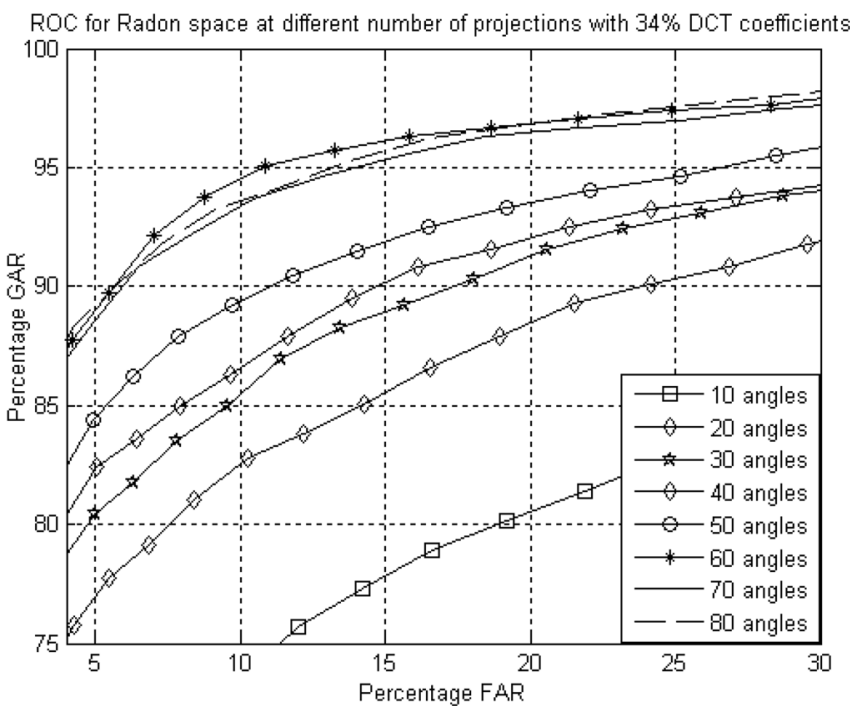

(b)

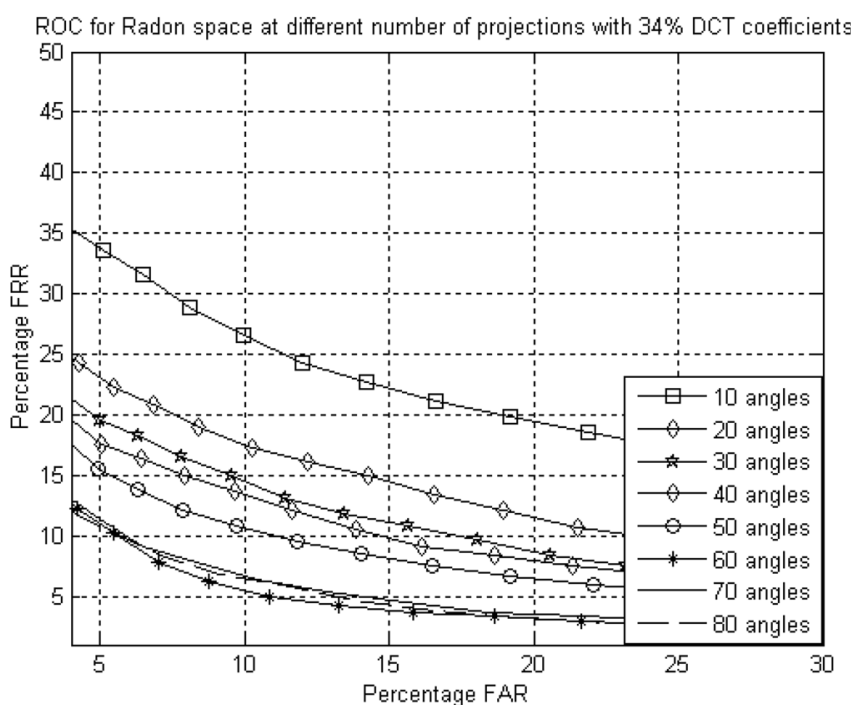

(d)

Figure 7. (a) Comparison of different percentages of DCT coefficients in terms of ROC (with GAR and FAR) on set1, (b) comparison of radon space at different number of projections with $34 \%$ DCT coefficients in terms of ROC (with GAR and FAR) on set1, (c) comparison of different percentages of DCT coefficients in terms of ROC (with FRR and FAR) on set1 and (d) comparison of radon space at different number of projections with $34 \%$ DCT coefficients in terms of ROC (with FRR and FAR).

\section{Proposed method}

The flow design of the proposed approach is shown in figure 1. It is built with functional blocks such as fingerprint image database, fingerprint image cropping, radon transform computation, DCT computation, estimation of region of interest, feature computation and feature database, similarity measure and matching.

\subsection{Fingerprint image database}

The performance of proposed method is evaluated on two databases namely (1) A: Db1_a of FVC2002 and (2) B: DB2_A of FVC2004. We used 500 images each of Databases A and B. Both the databases consist of images of 100 persons with 5 impressions of each person. Each image in Database A is of size $388 \times 374$. Each image in Database B is of size $328 \times 364$. The rotation-invariant property of 
Table 2. Comparison of number of angles used for radon transform in terms of GAR, FAR and FRR on set1.

\begin{tabular}{llll}
\hline \multirow{2}{*}{$\begin{array}{l}\text { No. of different angles used to compute } \\
\text { radon transform }\end{array}$} & \multicolumn{3}{c}{ Radon+DCT } \\
\cline { 2 - 4 } & GAR & FAR & FRR \\
\hline 10 & 81.4 & 21.89 & 18.6 \\
20 & 86.6 & 16.56 & 13.4 \\
30 & 88.24 & 13.45 & 11.76 \\
40 & 87.88 & 11.64 & 12.12 \\
50 & 89.24 & 9.74 & 10.76 \\
60 & $\mathbf{9 5 . 0 4}$ & $\mathbf{1 0 . 8 6}$ & $\mathbf{4 . 9 6}$ \\
70 & 94.6 & 12.82 & 5.4 \\
80 & 94.12 & 11.30 & 5.88 \\
90 & 94.2 & 10.64 & 5.8 \\
\hline
\end{tabular}

proposed method is tested on 30 rotated databases. These 30 rotated databases are created by rotating each database (A and B) by $30^{\circ}, 45^{\circ}, 60^{\circ}, 90^{\circ}, 120^{\circ}, 150^{\circ}, 210^{\circ}, 270^{\circ}$, $-15^{\circ},-20^{\circ},-30^{\circ},-45^{\circ},-60^{\circ},-120^{\circ}$ and $-150^{\circ}$.

\subsection{Fingerprint image cropping}

The use of whole fingerprint image for matching simply increases the number of computations and hence computational complexity. Hence, in this technique, the core point is positioned in fingerprint image and a little area in the region of core point is considered for feature extraction. Initially the point with maximum curvature called core point of fingerprint image is placed and an image of size $120 \times 120$ is cropped around the core point. Sample images from Databases A and B are shown in figure $2 a-d$ and $f-j$. Figure 2e shows the cropped image. Most of the images in both databases have rotation by less than or greater than $45^{\circ}$. Figure $3 \mathrm{a}$ shows the original image and figure $3 \mathrm{~b}$ shows the cropped image. Figure $3 \mathrm{c}, \mathrm{e}, \mathrm{g}$ and i shows, respectively, the $+30^{\circ},-30^{\circ}, 60^{\circ}$, and $-60^{\circ}$ in-plane-rotated images. Figure $3 \mathrm{~d}, \mathrm{f}, \mathrm{h}$ and $\mathrm{j}$ shows the cropped image from figure $3 \mathrm{c}, \mathrm{e}, \mathrm{g}$ and $\mathrm{i}$, respectively.

\subsection{Radon transform computation}

Before applying the radon transform the cropped image is enhanced using histogram equalization. The radon transform of cropped image is computed. The radon transform converts the rotation of an image into translation. Figure $4 \mathrm{a}$ shows the original image and figure $4 \mathrm{~b}$ and $\mathrm{c}$ shows the cropped image and its radon transform. Figure $4 d, f$ and $h$ shows the cropped image from the original image rotated by $30^{\circ}, 60^{\circ}$ and $90^{\circ}$, respectively. Figure $4 \mathrm{e}$, g and i shows the corresponding radon transform. In the radon transform, for the image rotated by $30^{\circ}$, correspondingly there is just translation by $30^{\circ}$ in radon space as shown in figure $4 \mathrm{c}$ and e. We can observe in figure $4 \mathrm{e}, \mathrm{g}$ and $\mathrm{i}$ that the effect of rotation of an image results in translation in radon space.
We considered radon space of 60 different angles in the step of 3 between $0^{\circ}$ and $180^{\circ}$. We experimentally proved that radon space of 60 different angles between $0^{\circ}$ and $180^{\circ}$ gives sufficient information for feature extraction. The increase in number of radon projections beyond 60 does not improve the system performance but contributes to computational complexity.

\subsection{DCT computation}

The translation-invariant features can be computed using 2D DCT of radon space. The important property of DCT is energy compaction as elaborated in $[32,33]$. The energy compaction property of DCT is illustrated by computing 1D DCT of radon transform at only one angle. Figure 5a shows the radon space of cropped image at angle $90^{\circ}$. Figure $5 \mathrm{~b}$ shows the 1D DCT of radon transform at angle $90^{\circ}$. Figure $5 \mathrm{~b}$ shows that most of the energy is concentrated in the first 50 DCT coefficients and others coefficients have negligible value. This shows the energy compaction property of DCT. 2D DCT is used for 60 angles of radon space shown in figure $5 \mathrm{c}$. Figure $5 \mathrm{~d}$ shows the 2D DCT of figure $5 \mathrm{c}$. In figure $5 \mathrm{~d}$, most of the energy is distributed near the top right corner.

\subsection{Estimation of region of interest}

Figure $5 \mathrm{~d}$ shows that most of the DCT coefficients have negligible magnitude. The DCT coefficients with negligible magnitude do not give good feature set. Hence we considered the DCT coefficients that have significant value for feature vector formation. In figure $5 \mathrm{~b}$, we can observe that most of the energy is concentrated near the top right corner. The region of interest is found by analysing six square portions near the top right corner of 2D DCT image of size $30 \times 30,40 \times 40,50 \times 50,60 \times 60,70 \times 70$ and $80 \times 80$ as shown in figure $6 \mathrm{a}-\mathrm{f}$. The analysis is performed in terms of GAR, FAR, FRR and ROC. Results show that the square portion of size $60 \times 60$ performs better in terms of GAR, FAR, FRR and ROC.

\subsection{Feature extraction}

Instead of using the DCT coefficients of square portion of size $60 \times 60$ as it is, we extracted features for compact representation of fingerprint image. To extract features, the square portion of size $60 \times 60$ is divided into $5 \times 5$ nonoverlapping blocks and standard deviation of each block is computed. The division of the square portion of size $60 \times$ 60 into $5 \times 5$ non-overlapping blocks gives totally 144 blocks. The feature vector of length 144 is obtained after computing standard deviation of each block. The feature vector of each database image is created and accumulated to obtain the feature vector of the database. The standard deviation is given by the equation 


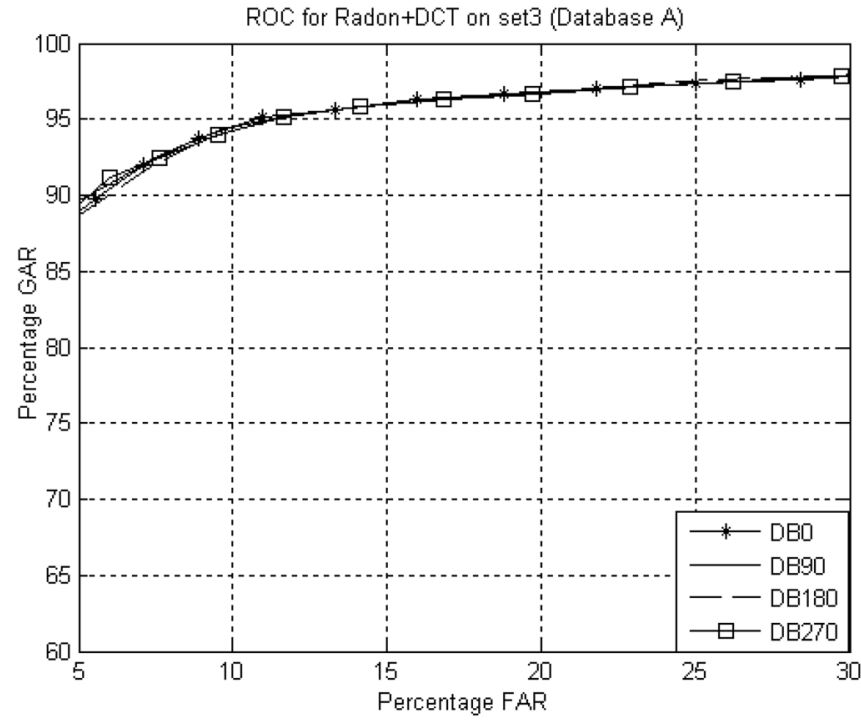

(a)

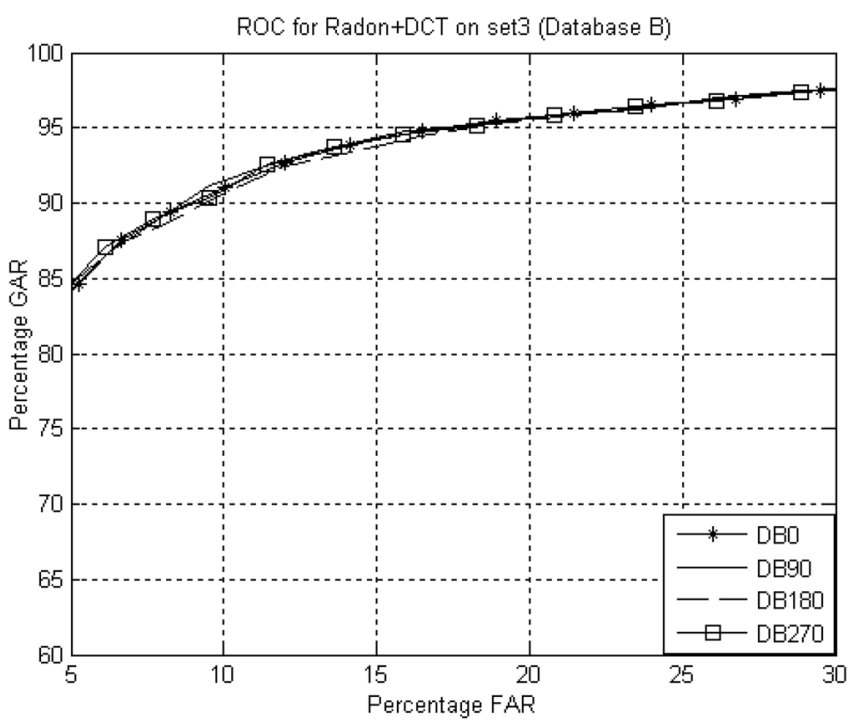

(c)

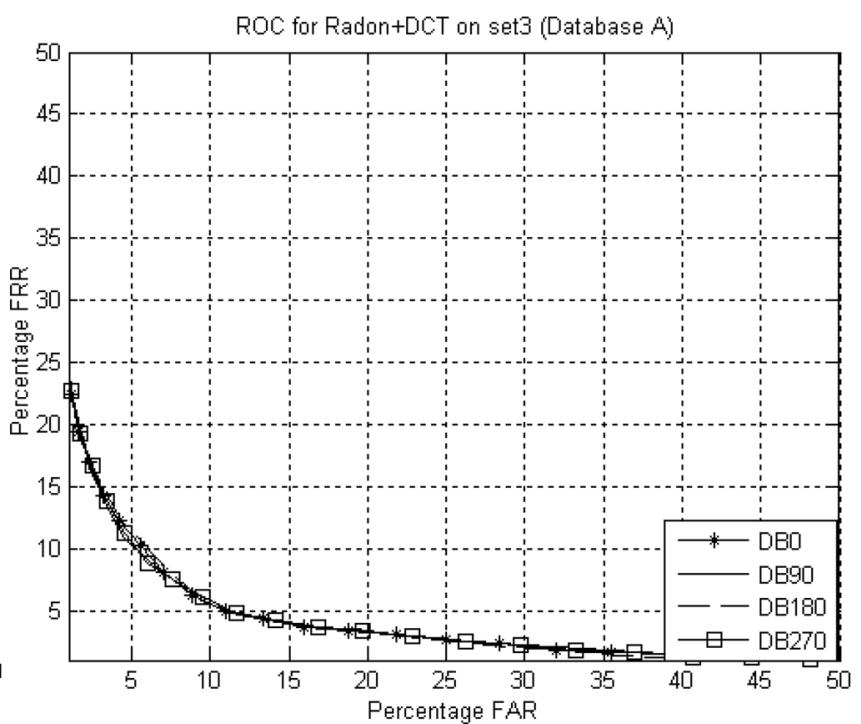

(b)

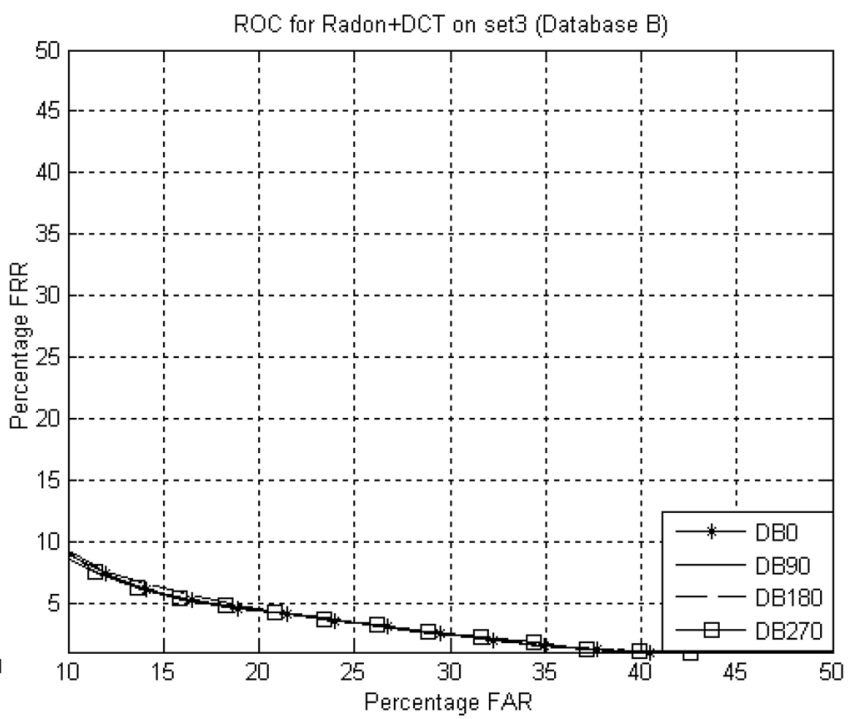

(d)

Figure 8. (a) Comparison of proposed method in terms of ROC (a) with GAR and FAR on set3 (Database A), (b) with FRR and FAR on set3 (Database A), (c) with GAR and FAR on set3 (Database B) and (d) with FRR and FAR on set3 (Database B).

$$
S=\sqrt{\frac{1}{N \times N} \sum_{i=0}^{N-1} \sum_{j=0}^{N-1}(b(i, j)-M)^{2}}
$$

where $b(i, j)$ denotes the pixel value of the block, $N \times N$ is the size of the block, $S$ is the standard deviation of the block and $M=\frac{1}{N \times N} \sum_{i=0}^{N-1} \sum_{j=0}^{N-1} b(i, j)$.

\subsection{Distance measure and matching}

Distances between the query image and database images are calculated to find the similarity between the query image and database images. A small distance between the query image and database image shows that the query image is more like the database image, and large distance between them shows the dissimilarity between the query image and database image. Euclidean distance is the square root of sum of the square difference in each dimension. For small variations in query image, the square difference increases the dissimilarity between the query image and database image. Hence we preferred the well-judged approach of calculating the sum of the absolute difference in each dimension rather than their squares, taken as a quantification of dissimilarity [34]. This distance metric, which considers the sum of the absolute difference for every dimension, is very often called the City Block or the 


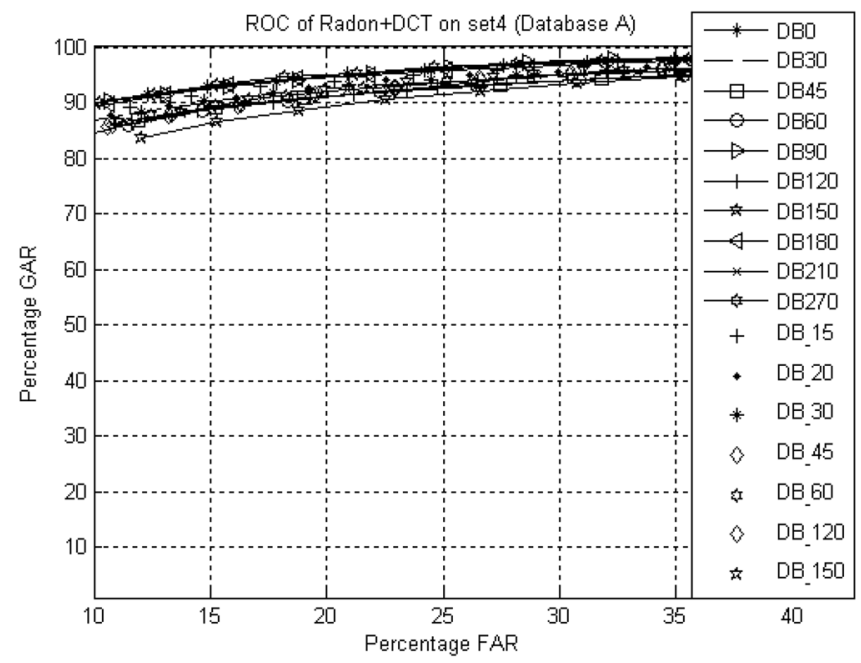

(a)

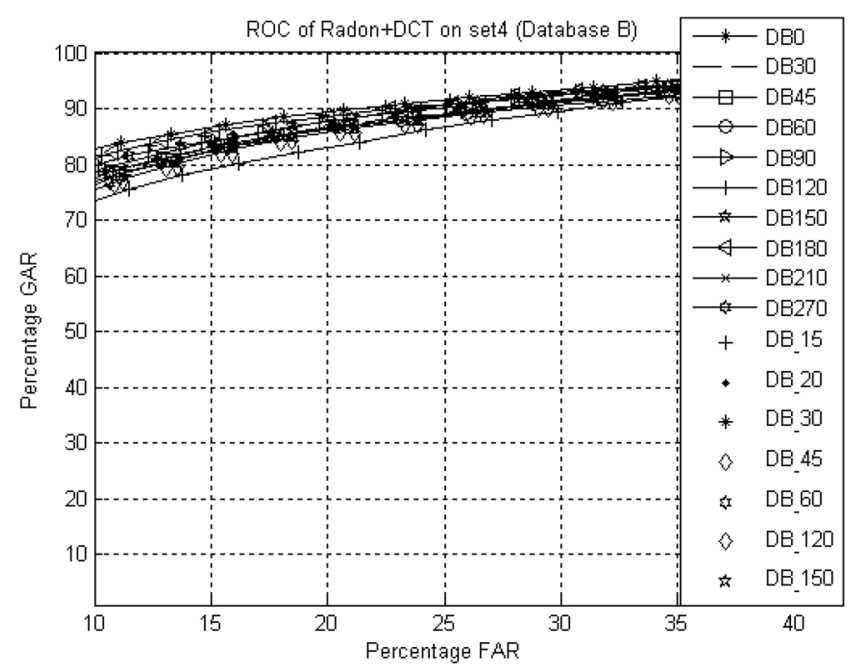

(c)

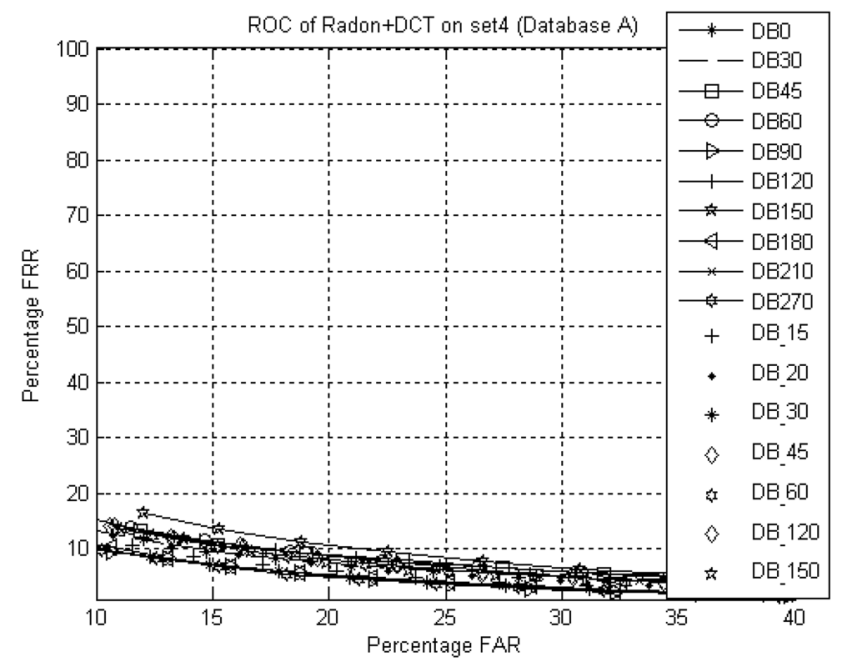

(b)

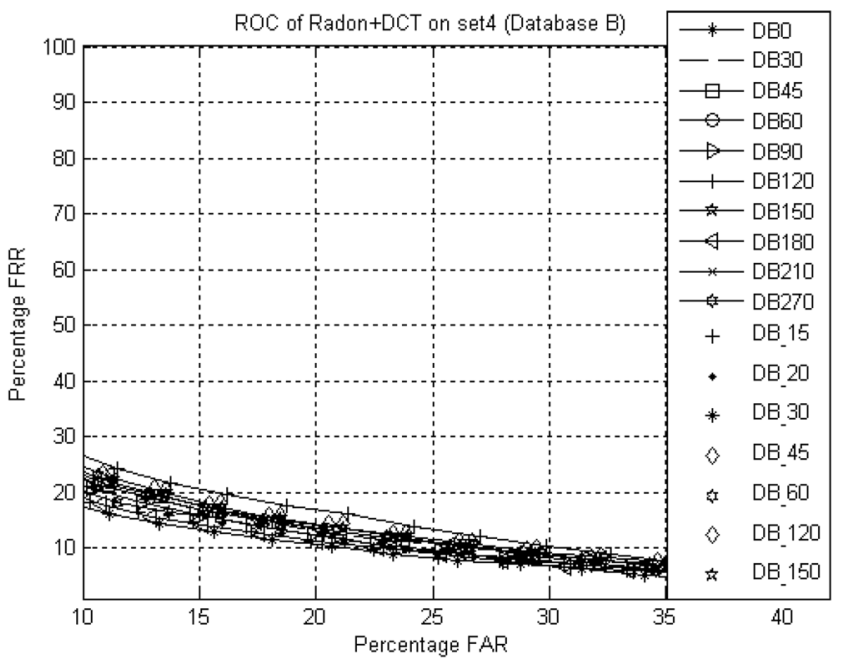

(d)

Figure 9. (a) Comparison of proposed method in terms of ROC (a) with GAR and FAR on set4 (Database A), (b) with FRR and FAR on set4 (Database A), (c) with GAR and FAR on set4 (Database B) and (d) with FRR and FAR on set4 (Database B).

Manhattan distance or $\mathrm{L}_{1}$ distance. The $\mathrm{L}_{1}$ distance metric for $\mathrm{N}$-dimensional feature vectors is defined by the equation

$$
d_{m}\left(F_{i}, F_{t}\right)=\sum_{j=1}^{N}\left|F_{i j}-F_{t j}\right|
$$

for $N$-dimensional feature vectors $F_{i}=\left(F_{i 1}, F_{i 2}, F_{i 3}, \ldots \ldots\right.$, $\left.F_{i N}\right)$ and $F_{t}=\left(F_{t 1}, F_{t 2}, F_{t 3}, \ldots \ldots, F_{t N}\right)$ where $F_{i}, F_{t}$ are the features of database image and query image, respectively. $N$ is the number of features in each feature vector.

After calculation of distances between the query image and database images, these distances are organized in an increasing order. The threshold is calculated by taking the average of top five distances. A value somewhat greater than the average of top five distances is selected as the final threshold for fingerprint matching.

\section{Experimental results}

The four metrics, namely GAR, FAR, FRR and ROC, equal error rate (EER) and recognition accuracy are considered for measurement of performance of our approach. These metrics are described briefly as follows.

a. FRR - the percentage of authentic persons rejected by the fingerprint recognition system is called FRR. It is represented by the equation

$$
F R R=\frac{\text { true claims rejected }}{\text { total true claims }} \times 100 .
$$

b. FAR - the percentage of impostors accepted by the fingerprint recognition system is called FAR. It is represented by the equation 


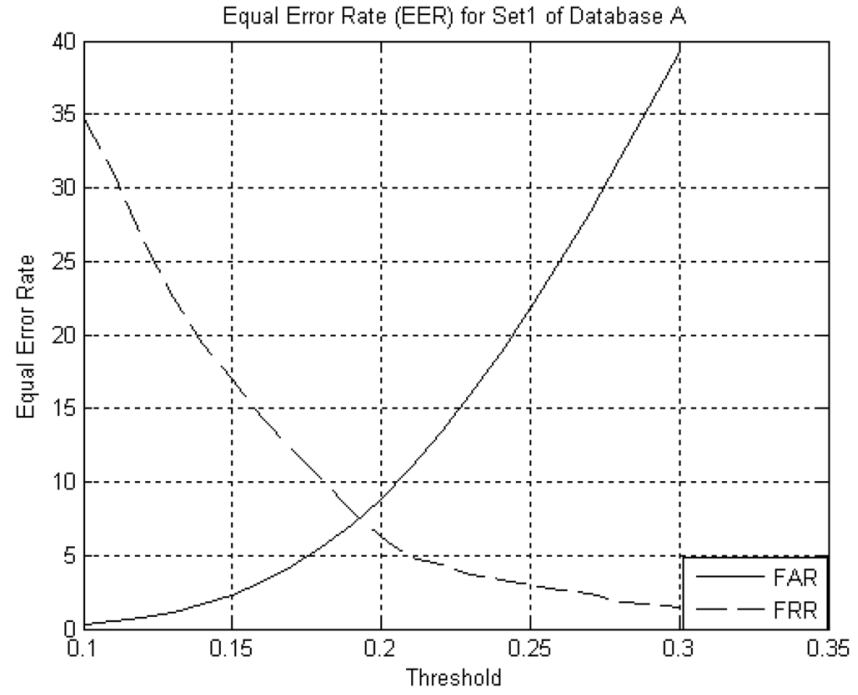

(a)

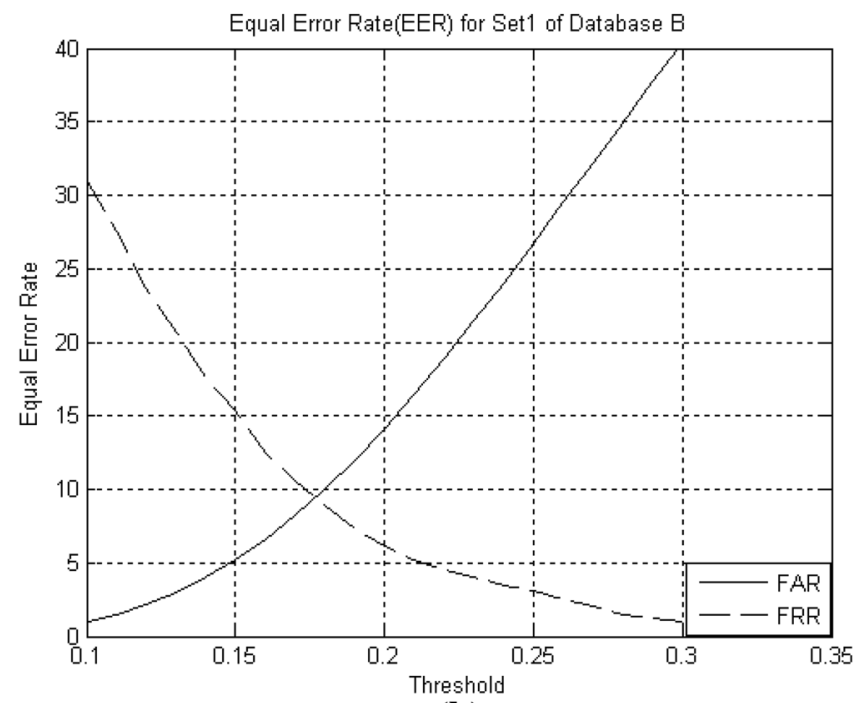

(b)

Figure 10. EER for set1: (a) Database A and (b) Database B.

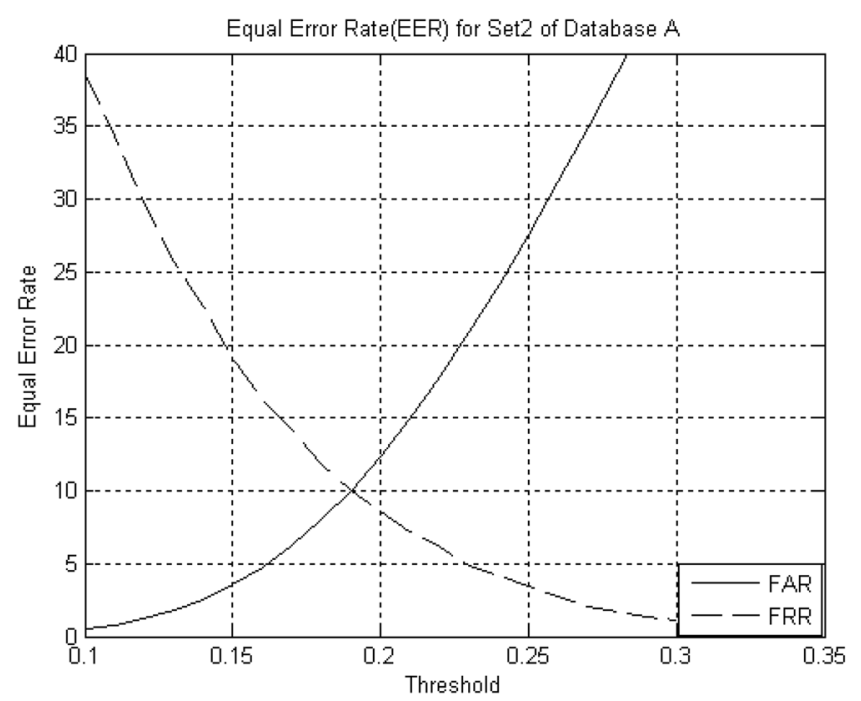

(a)

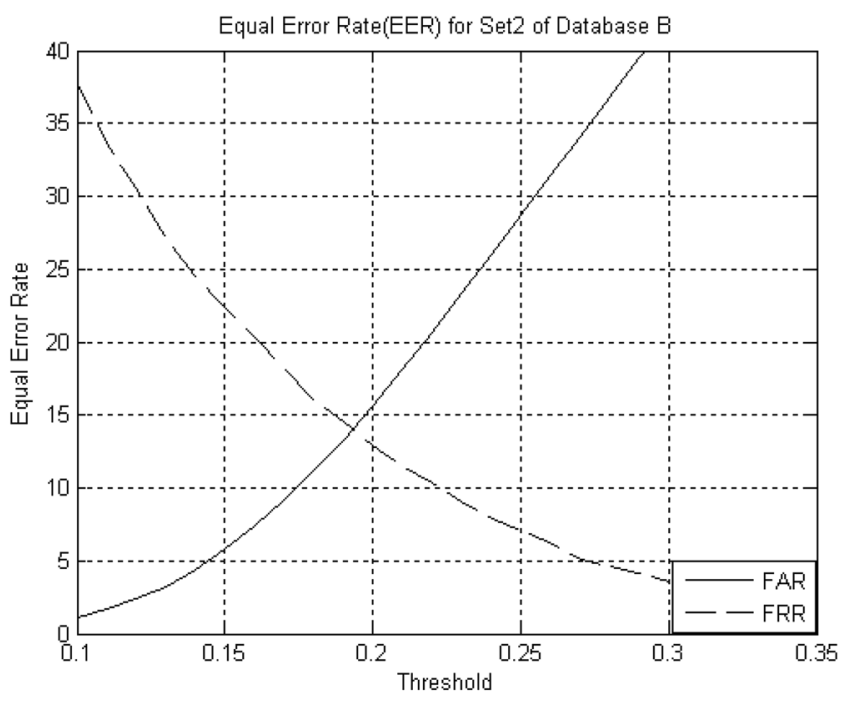

(b)

Figure 11. EER for set2: (a) Database A and (b) Database B.

$$
F A R=\frac{\text { imposter claims accepted }}{\text { total imposter claims }} \times 100 .
$$

c. GAR - the percentage of authentic persons accepted by the fingerprint recognition system is called as GAR. It is represented by the equation

$$
G A R=(1-F R R) \times 100 .
$$

d. ROC-it is a plot of GAR or FRR against FAR.

e. EER-it is defined as the rate at which both FAR and FRR errors are equal. It is represented by the equation

$$
\mathrm{EER}=\mathrm{FAR} \text { for which FAR }=\text { FRR. }
$$

f. Recognition accuracy-it is used to measure the performance of the fingerprint recognition system. It is represented by the equation

$$
\text { recognition accuracy }=\left(100-\frac{\text { FAR }+ \text { FRR }}{2}\right) .
$$

Experimental results are obtained using MATLAB 7 on a Pentium IV machine. 


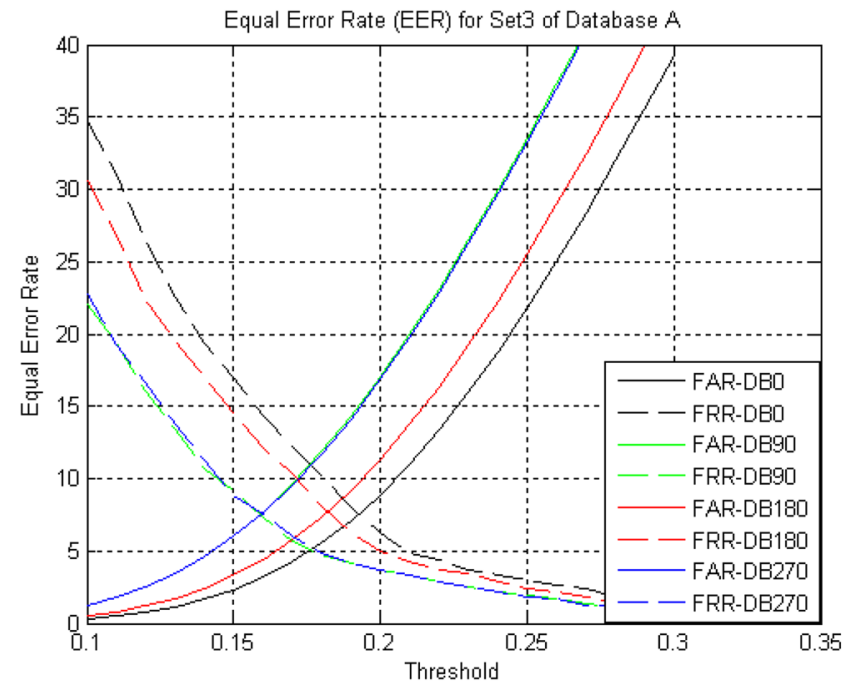

(a)

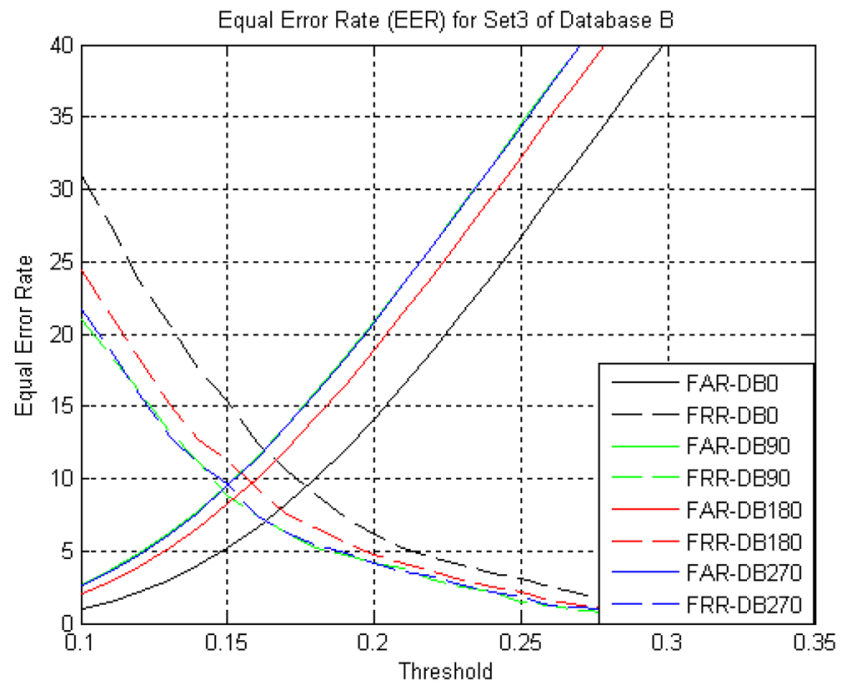

(b)

Figure 12. EER for set3: (a) Database A and (b) Database B.

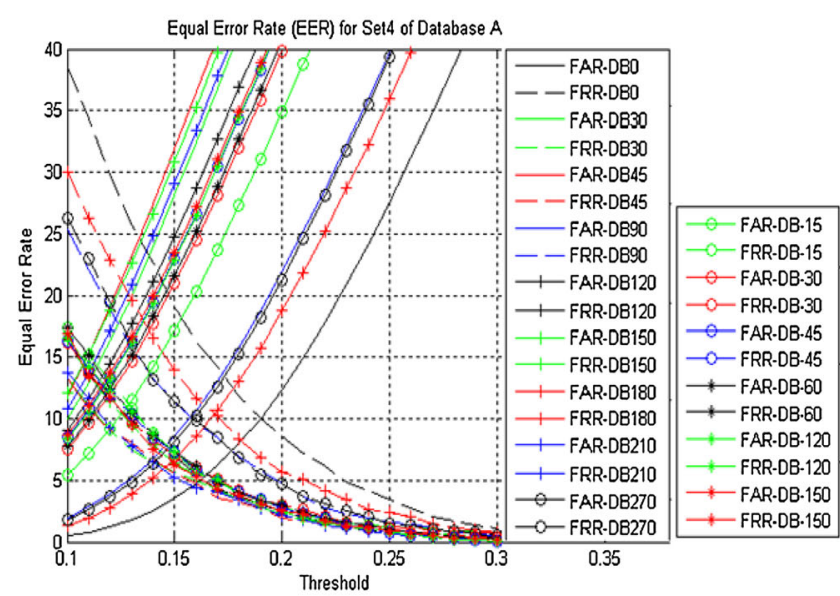

(a)

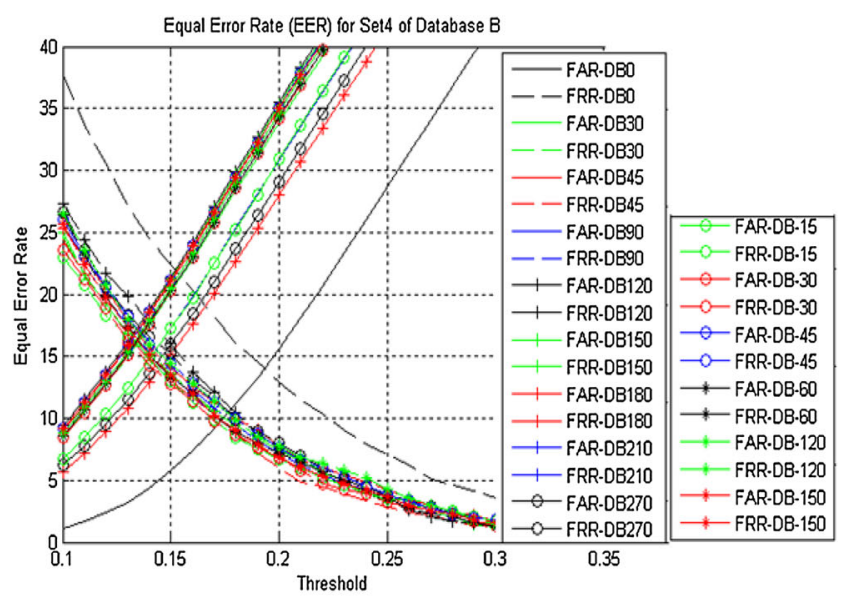

(b)

Figure 13. EER for set4: (a) Database A and (b) Database B.

\subsection{Formation of four sets of fingerprint image databases}

For extensive evaluation of the proposed method, four different sets of databases are formed using non-rotated Databases A, B and thirty rotated databases to prove the rotation-invariant property of proposed method.

a. Set1: set 1 consists of the non-rotated Databases A and B. In set 1 the core point is located manually for accuracy.

b. Set2: set 2 consists of the non-rotated Databases A and B. In set2 the core point is located using core point detection algorithm used in [2].

c. Set3: set3 consists of the three rotated Databases DB90, DB180 and DB270 of each Database A and B. In set3 the core point is located manually for accuracy. Most of the images in Databases A and B have rotation by more than or less than $45^{\circ}$. Due to this, these three major degrees of rotations are considered in this set.

d. Set4: set4 is formed by rotation of non-rotated Databases A and B. Fifteen rotated databases of each Database A and $\mathrm{B}$ are created by rotating the $\mathrm{Db} 1 \_$a database by $30^{\circ}$, $45^{\circ}, 60^{\circ}, 90^{\circ}, 120^{\circ}, 150^{\circ}, 210^{\circ}, 270^{\circ},-15^{\circ},-20^{\circ},-30^{\circ}$, $-45^{\circ},-60^{\circ},-120^{\circ}$ and $-150^{\circ}$. In set 4 the core point is located using the core point detection algorithm used in [2].

\subsection{Selection of optimum number of radon projections and DCT coefficients}

As discussed in section 3.5, the optimum numbers of radon projections are found out by evaluating radon space at 
different projections in terms of GAR, FAR and FRR. In the same way, optimum DCT coefficients are obtained by analysing the different low-frequency square regions of $2 \mathrm{D}$ DCT in terms of GAR, FAR and FRR. The performance of proposed method is evaluated in terms of GAR, FAR and FRR. Comparison of six square portions of 2D DCT of image is carried out in terms of GAR, FAR and FRR on set1. Table 1 shows the comparison of six square portions and corresponding percentage DCT coefficients in terms of GAR, FAR and FRR. Figure $7 \mathrm{a}$ and $\mathrm{c}$ shows the comparison of percentage DCT coefficients in terms of ROC with GAR, FAR and ROC with FRR, FAR, respectively. Results in table 1 and ROC shown in figure 7a, c show that the square portion of size $60 \times 60$ with $\sim 34 \%$ DCT coefficients gives better GAR at low FAR as compared with rest of the square portions. The increase in percentage DCT coefficients beyond $34 \%$ does not show any increase in GAR and decrease in FAR. Hence the square portion of size $60 \times 60$ with $\sim 34 \%$ DCT coefficients is considered as the optimum square portion or region of interest for feature extraction.

The optimum number of angles for radon space is computed by analysing the radon space for $10,20,30,40$, 50, 60, 70, 80 and 90 different angles between $0^{\circ}$ and $180^{\circ}$ in terms of GAR, FAR and FRR. Table 2 shows comparison of angles used for radon transform in terms of GAR, FAR and FRR on set1. Figure $7 b$ and $d$ shows comparison of number of projections used for radon transform in terms of ROC with GAR, FAR and ROC with FRR, FAR, respectively. Results in table 2 and ROC shown in figure $7 \mathrm{~b}$, d illustrate that the radon space for 60 different angles between $0^{\circ}$ and $180^{\circ}$ gives better GAR at low FAR and FRR as compared with the other angles. Increase in number of angles beyond 60 for radon space does not show an increase in GAR and decrease in FAR as well as in FRR. Hence, 60 (angles between $0^{\circ}$ and $180^{\circ}$ ) is considered as the optimum number of angles for feature extraction. Tables 1 and 2 show that 60 different angles for radon space with $\sim 34 \%$ DCT coefficients gives better performance. The proposed method gives $70 \%$ GAR at $\sim 0 \%$ FAR and $30 \%$ FRR, and $\sim 95 \%$ GAR at $\sim 10 \%$ FAR and $5 \%$ FRR.

\subsection{Assessment of rotation-invariant radon $+D C T$ features based on ROC}

Figures $8 \mathrm{a}-\mathrm{d}$ and $9 \mathrm{a}-\mathrm{d}$ show comparison of the proposed method in terms of ROC (with GAR and FAR, with FRR and FAR) on Databases A, B of set 3 and set4. In figure 8a, $\mathrm{b}$ and $\mathrm{c}, \mathrm{d}$, the ROCs of rotated and non-rotated databases of set 3 are exactly equal. Set 4 consists of 15 in-plane-rotated databases of Database $\mathrm{A}$ and 15 in-plane-rotated databases of Database B. The ROCs in figure 9a and b on 15 in-plane-rotated databases (Database A) are almost overlapping. Also the ROCs in figure $9 \mathrm{c}$ and $\mathrm{d}$ on 15 in-
Table 3. EER (FAR=FRR) for the proposed method.

\begin{tabular}{|c|c|c|c|c|c|}
\hline \multirow[b]{2}{*}{ Set } & \multirow{2}{*}{\multicolumn{2}{|c|}{ Database }} & \multirow[b]{2}{*}{ GAR $(\%)$} & \multicolumn{2}{|c|}{ EER } \\
\hline & & & & FAR $(\%)$ & FRR $(\%)$ \\
\hline \multirow[t]{2}{*}{ Set1 } & & A & 92.44 & 7.56 & 7.56 \\
\hline & & B & 90.53 & 9.47 & 9.47 \\
\hline \multirow[t]{2}{*}{ Set2 } & & A & 90 & 10 & 10 \\
\hline & & B & 86 & 14 & 14 \\
\hline \multirow[t]{8}{*}{ Set3 } & A & DB0 & 92.44 & 7.56 & 7.56 \\
\hline & & DB90 & 92.48 & 7.52 & 7.52 \\
\hline & & DB180 & 92.28 & 7.72 & 7.72 \\
\hline & & DB270 & 92.44 & 7.56 & 7.56 \\
\hline & B & DB0 & 90.53 & 9.47 & 9.47 \\
\hline & & DB90 & 90.75 & 9.25 & 9.25 \\
\hline & & DB180 & 90.34 & 9.66 & 9.66 \\
\hline & & DB270 & 90.43 & 9.57 & 9.57 \\
\hline
\end{tabular}

Table 4. Comparison of the proposed method with existing methods in terms of GAR, FAR and FRR on set1.

\begin{tabular}{|c|c|c|c|c|}
\hline Method & Database & $\begin{array}{l}\text { Accuracy/ } \\
\text { GAR (\%) }\end{array}$ & $\begin{array}{c}\text { FAR } \\
(\%)\end{array}$ & $\begin{array}{l}\text { FRR } \\
(\%)\end{array}$ \\
\hline $\begin{array}{l}\text { Block } \\
\text { orientation } \\
\text { features [26] }\end{array}$ & A & 93 & 18 & 7 \\
\hline \multirow{2}{*}{$\begin{array}{l}\text { Local binary } \\
\text { pattern }[35]\end{array}$} & A & 92.10 & - & - \\
\hline & B & 91.23 & - & - \\
\hline \multirow{2}{*}{$\begin{array}{l}\text { Proposed } \\
\text { method }\end{array}$} & A & 95.04 & 10.86 & 4.96 \\
\hline & $\begin{array}{c}\text { B } \\
\text { Comparison in } \\
\text { terms EER } \\
(\mathrm{FAR}=\mathrm{FRR})\end{array}$ & 94.84 & 16.46 & 5.16 \\
\hline $\begin{array}{l}\text { Minutiae disk } \\
\text { code [10] }\end{array}$ & $\mathrm{A}$ & 82.3 & 17.70 & 17.70 \\
\hline $\begin{array}{l}\text { Minutiae } \\
\text { cylinder- } \\
\text { code [36] }\end{array}$ & A & 89.61 & 10.39 & 10.39 \\
\hline \multirow{2}{*}{$\begin{array}{l}\text { Proposed } \\
\text { method }\end{array}$} & A & 92.44 & 7.56 & 7.56 \\
\hline & B & 90.53 & 9.47 & 9.47 \\
\hline
\end{tabular}

plane-rotated databases (Database B) are almost overlapping. The ROC on rotated databases of set 3 and set 4 proves the rotation-invariant property of proposed method.

\subsection{Assessment of rotation-invariant radon $+D C T$ features based on EER, GAR, FAR and FRR}

Figures 10, 11, 12 and 13 show the threshold versus FAR, FRR plot for the set 1 , set 2 , set 3 and set 4 databases. Table 3 shows the EER for the proposed feature extraction method. Figures 12 and 13 show that the EERs for rotated and non- 
rotated databases are almost equal. Figures 12 and 13 and EER values presented in table 3 prove the rotation-invariant property of proposed method.

Table 4 shows comparison of the proposed method with the existing methods in terms of GAR, FAR, FRR and EER. EER metric is used in $[10,36]$ for performance evaluation of their method. In [26, 35], accuracy, GAR, FAR and FRR metrics are adopted for measurement of the performance of their method. Hence, in table IV, we provide the comparative analysis in terms of GAR, FAR, FRR and EER. Results in table 4 show the better performance of the proposed method over existing methods.

\section{Conclusion}

In this research work, we have demonstrated new rotationinvariant features using radon transform and DCT for fingerprint matching. Rotation invariance is achieved with a two-stage approach. In the first stage, radon transform converts rotation of an image into translation and in the second stage, translation invariance is achieved using DCT. Rotation invariance of the proposed method is consistently proved on non-rotated and rotated versions of standard databases, namely FVC2002 and FVC2004, in terms of GAR, FAR and ROC. The proposed method outperforms over the existing rotation-invariant feature extraction method [26] and a few more recent methods [10, 35, 36] for fingerprint recognition. The proposed method has dual advantages over earlier approach. Firstly, it is rotation and translation invariant. Secondly, it uses only 34\% DCT coefficients to form a compact feature vector, which significantly reduces the feature extraction and matching time. This is the key requirement of an automatic fingerprint identification system (AFIS). In future, the proposed method can be used for other pattern recognition applications like object detection, classification of medical images and rotation-invariant texture classification.

\section{References}

[1] Maltoni D, Maio D, Jain A and Prabhakar S 2003 Handbook of fingerprint recognition. New York: Springer

[2] Jain A, Prabhakar S, Hong L and Pankanti S 2000 Filterbankbased fingerprint matching. IEEE Trans. Image Process. 9: 846-859

[3] Bharkad S and Kokare M 2012 Fingerprint matching using M band wavelet transform. In: Proceedings of the IEEE Conference on Advances in Engineering, Science and Management (ICAESM), pp. 26-32

[4] Bharkad S and Kokare M 2013 Fingerprint matching using discrete wavelet packet transform. In: Proceedings of the IEEE Conference on Advanced Computing (AC), pp. $1183-1188$
[5] Bharkad S and Kokare M 2012 Rotated wavelet filter based fingerprint matching. Int. J. Pattern Recogn. Artif. Intell. 26(3): 1-21

[6] Bazen A and Gerez S 2003 Fingerprint matching by thinplate spline modeling of elastic deformations. Pattern Recogn. 36(8): 1859-1867

[7] Jain A, Hong L and Bolle R 1997 On-line fingerprint verification. IEEE Trans. Pattern Anal. Mach. Intell. 19: 302-314

[8] Bharkad S and Kokare M 2011 Fingerprint identification: ideas, influences, and trends of the new age. In: Pattern recognition, machine intelligence and biometrics (PRMI), Chapter 17, Springer, pp. 417-455

[9] Barman S, Chattopadhyay S, Samanta D, Bag S and Show G 2014 An efficient fingerprint matching approach based on minutiae to minutiae distance using indexing with effectively lower time complexity. In: Proceedings of the IEEE Conference on Information Technology (ICIT), pp. 179-183

[10] Chu T T and Chiu C T 2016 A cost-effective minutiae disk code for fingerprint recognition and its implementation. In: Proceedings of the IEEE Conference on Acoustic, Speech and Signal Processing (ICASSP), pp. 981-985

[11] Jiang L, Zhao T, Bai C, Yong A and Wu M 2016 Direct fingerprint minutiae extraction approach based on convolutional neural networks. In: Proceedings of the IEEE Conference on Neural Networks (IJCNN), pp. 571-578

[12] Nagaty K 2004 An energy-based fingerprint matching system. In: Proceedings of the IEEE Conference on Consumer Communications and Networking, pp. 706-709

[13] Jain A, Prabhakar S, Hong L and Pankanti S 1999 FingerCode: a filterbank for fingerprint representation and matching. In: Proceedings of the IEEE Conference on Computer Vision and Pattern Recognition, pp. 187-193

[14] Ross A, Jain A and Reisman J 2002 A hybrid fingerprint matcher. In: Proceedings of the IEEE, pp. 795-798

[15] Horton M, Meenen P, Adhami R and Cox P 2002 The costs and benefits of using complex 2D Gabor filter in a filter based fingerprint matching system. In: Proceedings of the IEEE Conference on System Theory, pp. 171-175

[16] Munir M and Javed M 2005 Fingerprint matching using ridge patterns. In: Proceedings of the IEEE Conference on Information and Communication Technology, pp. 116-120

[17] Bharkad S and Kokare M 2012 Hartley transform based fingerprint matching. Int. J. Inf. Process. 8(1): 85-100

[18] Baldi P and Chauvin Y 1993 Neural networks for fingerprint recognition. Neural Comput. 5(3): 402-418

[19] Song Q, Liu X and Yang L 2015 The random forest classifier applied in droplet fingerprint recognition. In: Proceedings of the IEEE Conference on Fuzzy Systems and Knowledge Discovery (FSKD), pp. 722-726

[20] Gu F, Wang Y and Cheung Y 2014 A supervised correlation analysis for score-level calibration of cross-device fingerprint recognition. In: Proceedings of the IEEE Conference on Systems, Man, and Cybernetics, pp. 1165-1170

[21] Jain A K, Arora S S, Cao K, Best-Rowden L and Bhatnagar A 2016 Fingerprint recognition of young children. IEEE Trans. Inf. Forens. Secur. 12(7): 1501-1514

[22] Ctirad S and Christoph B 2014 Presentation attack detection methods for fingerprint recognition systems: a survey. IET Biometr. 3(4): 219-233 
[23] Yu X, Xiong Q, Luo Y, Wang N, Wang L, Tey H L and Liu L 2017 Contrast enhanced subsurface fingerprint detection using high-speed optical coherence tomography. IEEE Photon. Technol. Lett. 29(1): 70-73

[24] Kim W 2017 Fingerprint liveness detection using local coherence patterns. IEEE Signal Process. Lett. 24(1): 51-55

[25] Kumar A and Kwong C 2015 Towards contactless, low-cost and accurate 3D fingerprint identification. IEEE Trans. Pattern Anal. Mach. Intell. 37(3): 681-696

[26] Kulkarni J V, Patil B D and Holambe R S 2006 Orientation feature for fingerprint matching. Pattern Recogn. 39(8): 1551-1554

[27] Sandhan T, Chang H J and Choi J Y 2013 Abstracted radon profiles for fingerprint recognition. In: Proceedings of the IEEE Conference on Image Processing, pp. 4156-4160

[28] Ray T and Dutta P K 2014 Texture classification by Rotational Invariant DCT Masks (RIDCTM) features. In: Proceedings of the IEEE Conference on Advances in Computing, Communications and Informatics (ICACCI), pp. 2041-2044

[29] Jadhav D V and Holambe R S 2009 Feature extraction using Radon and wavelet transforms with application to face recognition. Neurocomputing 72(7-9): 1951-1959
[30] Ajmera P K, Jadhav D V and Holambe R S 2011 Textindependent speaker identification using Radon and discrete cosine transforms based features from speech spectrogram. Pattern Recogn. 44(10-11): 2749-2759

[31] Toft P 1996 The Radon transform: theory and implementation. Ph.D. Thesis, Technical University of Denmark

[32] Gonzalez R C and Woods R E 1990 Digital image processing. 2nd edn, Upper Saddle River, New Jersey: Pearson Education

[33] Jain A 1989 Fundamentals of digital image processing. Englewood Cliffs, NJ: Prentice-Hall

[34] Bharkad S and Kokare M 2011 Performance evaluation of distance metrics: application to fingerprint recognition. Int. J. Pattern Recogn. Artif. Intell. 25(6): 777-806

[35] Gowthami A T and Mamatha H R 2015 Fingerprint recognition using zone based linear binary patterns. Proc. Comput. Sci. 58: 552-557

[36] Cappelli R, Ferrara M and Maltoni D 2010 Minutiae cylinder-code: a new representation and matching technique for fingerprint recognition. IEEE Trans. Pattern Anal. Mach. Intell. 32(12): 2128-2141 